\title{
La spéculation sur les matières premières ${ }^{1}$
}

\author{
Paul JORION \\ Sociologue et anthropologue \\ <paul_jorion@msn.com>
}

\begin{abstract}
What part has to be indebted to the financial speculation in the recent food an energy price inflation? Informations on this controversial question may be found through the works of different commissions of the US Senate. Particularly lights have been bring upon the mechanisms by which new speculators, made "investments" in the commodities futures market, and behave differently from the traditional speculators that have always existed in this marketplace.

Key words: commodities and oil price, financial speculation
\end{abstract}

marchés organisés de futures où ces fonds institutionnels investissaient, le même envol des prix que pour le blé ou le maïs pour lesquels de tels contrats à terme existent bien sur un marché à terme organisé.

Dans les jours qui suivirent, au lieu d'adopter un profil bas, ces fonds institutionnels crurent bon de pousser des cris d'orfraie, accumulant déclarations officielles et communiqués de presse, mettant fin à l'incertitude en faisant naïvement l'aveu de leur culpabilité.

Voilà donc où nous étions rendus : la nouvelle génération de méchants, désignés à la vindicte publique, n'étaient autres que ceux qui s'efforçaient désespérément de protéger la retraite des baby-boomers, l'avenir de la recherche et les bourses d'étudiants.

Le mécanisme qui avait conduit là était bien entendu très simple: les capitaux ayant $d^{\prime}$ abord fui les prêts hypothécaires subprime, et le feu s'étant propagé aux Asset-Backed Securities, puis aux Collateralized-Debt Obligations, suivies des Structured Investment Vehicles, et enfin aux obligations municipales, tous secteurs désormais sinistrés, cherchèrent à se placer sur les rares produits financiers ayant survécu à la peste des mois précédents et dont le nombre se réduisait de jour en jour, provoquant une bulle financière affectant l'ensemble des marchés mondiaux de matières premières.

\section{Quelques postulats}

Cet article est extrait du livre La Crise. Des subprimes au séisme financier planétaire, chapitre VIII, à paraître aux Editions Fayard (novembre 2008).

Paul Jorion, sociologue et anthropologue, a travaillé durant les dix dernières années dans le milieu bancaire américain en tant que spécialiste de la formation des prix. II a publié récemment L'implosion. La finance contre l'économie (Fayard: 2008) et Vers la crise du capitalisme américain? (La Découverte: 2007). http://www.pauljorion.com/blog/
Les arguments avancés par ceux qui niaient que les prix atteints alors par les matières premières étaient essentiellement spéculatifs, étaient identiques à ceux qu'avaient utilisés ceux qui niaient la bulle de l'immobilier résidentiel américain.

Une analyse était donc nécessaire, en dépit de l'absence d'une théorie de «science économi- que » pouvant prouver de manière irréfutable l'existence des bulles financières. Quelques postulats pouvaient servir de point de départ : 1. Le prix des matières premières nonrenouvelables (pétrole) et le prix des matières premières renouvelables (céréales) sont deux affaires distinctes.

2. De multiples facteurs jouent dans la détermination du prix d'une matière première. Mettre en évidence des facteurs qui influent sur l'offre et la demande, n'exclut pas qu'un prix puisse être essentiellement spéculatif.

3. Le prix d'une matière première révèle comme tout prix - le rapport de forces entre acheteurs et vendeurs. $Y$ a-t-il dans ce rapport de forces une « bonne » composante, qui refléterait l'offre et la demande, et une " mauvaise " composante que l'on pourrait éliminer? C'est la question que pose indirectement le projet de loi Stop Excessive Energy Speculation Act of $2008^{2}$ déposé le 15 juillet 2008 devant le Sénat américain, qui introduit la notion de « spéculation excessive ».

4. Y a-t-il une raison quelconque qui justifie la présence sur le marché à terme d'une matière première $d$ 'intervenants qui $n^{\prime}$ ont aucune capacité effective à livrer la marchandise ou à en prendre livraison à maturité ? L'argument, souvent proposé, de « liquidité », dont les spéculateurs seraient les dispensateurs n'a de sens que s'il y a capacité effective à livrer ou à prendre livraison des marchandises sur lesquelles portent les contrats à terme.

\section{Définir la spéculation}

Le prix d'une matière première est «spéculatif », si celui-ci est significativement supérieur à

\footnotetext{
$\overline{2}$ http://www.dpc.senate.gov/dpcnew.cfm?doc_name $=\mid \mathrm{b}-110-2-117$
} 
celui calculé de manière " additive ", en faisant la somme du prix des composants («fondamentaux »).

On peut bien entendu parler de « spéculation » au sens large et utiliser le terme pour caractériser quiconque essaye de gagner de l'argent sur les marchés. Dans ce cas-là, un manipulateur de l'offre - ou de la demande - est effectivement un « spéculateur »; quelqu'un qui commet un délit d'initié est lui aussi un spéculateur " au sens large ». Or, il faut opposer les facteurs constitutifs du prix qui relèvent de l'offre et de la demande et ceux qui relèvent de la spéculation « au sens étroit », qui est elle une simple manipulation du prix, absolument indépendante de la marchandise. Celui qui voudrait manipuler l'offre de pétrole a besoin de barils, $d^{\prime}$ une aire de stockage, etc., alors que celui qui spécule sur le pétrole, n'a même pas besoin de savoir ce qu'est le pétrole : la seule chose qu'il lui faille connaître, c'est si son prix est en train de monter ou s'il est en train de baisser.

Voici un exemple de spéculation, emprunté à un livre de Jack Schwager [1]. II interroge un trader appelé Monroe Trout :

"Par exemple, si je suis long de mille contrats futures Standard \& Poor's 500 (contrat à terme sur l'un des indices boursiers de la bourse principale de New York: le New York Stock Exchange) et qu'il est onze heures trente à l'heure de Chicago, je vais probablement vouloir introduire une sorte d'ordre d'achat de volume décroissant, comme acheter dix lots chaque fois que le marché baisse d'un tick (le tick vaut cinq centimes sur ce marché), pour tenir le marché dans ma direction. II ne faudra pas un très grand nombre de contrats à ce moment-là de la journée pour soutenir le marché puisqu'il n'y a que peu de contrats échangés [il est $12: 30$ hà New York et les négociateurs déjeunent] » (in Schwager 1992 : 155).

La tactique de Monroe Trout est une tactique de manipulation du prix et à ce titre, elle est applicable à n'importe quel marché, quel que soit le produit qui y est vendu.

Si l'on veut absolument situer la spéculation sur les marchés à terme de matières premières dans un cadre de référence d'offre et de demande, on peut faire comme le fait Mike Masters : dire que les spéculateurs constituent des « stocks virtuels».

\section{Le prix du pétrole}

Le pétrole est désormais en quantité limitée, et l'agriculture contemporaine repose en grande partie sur les dérivés des produits pétroliers. Les spéculateurs tirent parti de ces deux évidences et agitent désormais le spectre du « pic pétrolier » afin de détourner l'attention du public de leurs agissements. Le " pic pétrolier» existe certainement, il s'agit cependant d'une autre histoire, dont il convient de parler séparément.
En décembre 2007, Mr. Fadel Cheit de Oppenheimer \& Co., fut appelé à témoigner devant le comité Energy and Natural Resources du Sénat américain, en sa qualité d'expert sur la formation du prix de l'énergie. De 2004 à 2007, l'agence de presse Bloomberg lui a décerné son prix du meilleur analyste du marché pétrolier. Mr. Gheit affirma entre autres devant les membres de cette commission que :

"La mondialisation des marchés financiers, la facilité avec laquelle il est possible d'opérer, la vitesse à laquelle des fonds considérables peuvent circuler, la surabondance d'information, les tensions internationales accrues, ont créé un environnement idéal pour une spéculation excessive sur les marchés mondiaux du pétrole. "

Cette « spéculation excessive » est encouragée selon lui par l'absence de consensus quant aux fondamentaux du marché pétrolier :

"Personne n'est à même de prédire exactement ou de manière cohérente le prix du pétrole : ni les compagnies pétrolières, ni les gouvernements, ni les investisseurs sur la place de Wall Street. Cette absence de prévision fiable a créé un vide dans lequel se sont engouffrés des acteurs financiers à I'horizon d'investissement ultra-court, ce qui a contribué à une volatilité accrue des prix. "

Cette volatilité accrue $a$, à son tour, influencé les fondamentaux:

"La volatilité elle-même a un effet négatif sur l'industrie pétrolière parce qu'elle engendre l'incertitude et opère une distorsion des fondamentaux du marché, ce qui débouche sur des décisions d'investissement inappropriées quant à la constitution d'une offre qui correspondrait de manière adéquate à la demande globale en produits énergétiques. »

Gheit en concluait que le prix à terme du pétrole brut au moment de sa déposition: 98 dollars, était double de ce qu'il serait en l'absence d'un impact de la « spéculation excessive », prix à comparer aux 147 dollars qui furent atteints quand le prix culmina le 11 juillet 2008.

Gheit ajoutait que

"... les spéculateurs sur le marché pétrolier utilisent les données publiées hebdomadairement par I'Energy Information Administration pour manipuler les prix en vue d'un gain à court terme. Les spéculateurs agitent les chiffres signalant une baisse des inventaires pour répandre la peur quant à d'éventuels déficits, alors que cette baisse indique précisément le contraire : une adaptation efficiente des capitaux dans un environnement de prix élevés. »

Gheit proposait enfin aux sénateurs de prendre un certain nombre de mesures:

- Porter à $50 \%$ de la valeur du contrat la marge sur les opérations à terme (qui se monte à moins de $10 \%$ à l'heure actuelle).

- Limiter le nombre de contrats par compte individuel.
- Fixer une période minimale durant laquelle un contrat doit être conservé.

- Prévenir les conflits d'intérêt pour les institutions financières impliquées.

- Imposer des pénalités sévères pour les contrevenants, assorties d'un emprisonnement minimum.

En décembre, la déposition de Mr. Fadel Gheit passa inaperçue. Les choses changèrent et il fut ensuite abondamment cité dans la presse: "Pourquoi le prix peut-il grimper de $5 \%$ dans la journée, pour retomber un peu plus tard, s'il s'agit de fondamentaux?", demandait-il. A quoi il ajoutait : "Il existe une déconnexion totale entre le prix et l'offre et la demande. Les nouvelles règles sont : il n'y a plus de règles" ".

L'événement intervenu entre-temps, et qui contribua à le mettre à la une, ce fut la déposition de Michael Masters, le 20 mai, devant le comité des Homeland Security et Governmental Affairs.

\section{Les marchés à terme}

Si l'on veut comprendre ce qui survivra à la crise présente et ce qu'elle engloutira, une distinction essentielle doit être faite entre un risque financier auquel on est exposé à son corps défendant et contre lequel il est légitime de se protéger, et un risque auquel on s'expose de manière délibérée et qui constitue un danger, non seulement pour soi mais pour tous ceux qui dépendent de soi directement ou indirectement. Le risque inévitable est dicté par les circonstances, par exemple par l'ensemble des facteurs naturels ou d'ordre humain susceptibles d'influer sur le volume final d'une moisson. Le risque évitable est lui un pari que l'on fait, où l'on s'expose de manière librement consentie à une perte éventuelle en vue d'un gain incertain.

Les marchés à terme furent inventés pour permettre à des intervenants, l'un qui livrera et l'autre qui prendra livraison, de déterminer aujourd'hui à quel prix aura lieu une transaction qui prendra place ultérieurement. En fixant le prix dès maintenant, les deux parties, acheteur et vendeur, se protègent contre les aléas de la variation du prix susceptibles d'intervenir d'ici la livraison. À la maturité du contrat, au jour préétabli de la livraison, le prix à terme et le prix « spot », le prix au comptant, auront convergé jusqu'à - en principe - coïncider. Je dis «en principe» parce que cette coïncidence n'est plus nécessairement constatée aujourd'hui en raison de l'intense spéculation qui s'observe sur ces marchés.

Les contrats à terme avaient lieu traditionnellement «de gré à gré » (en anglais Over the Counter ou OTC) entre deux parties qui se mettaient d'accord sur les termes de la vente 
de manière anticipée à la transaction proprement dite, à savoir au transfert effectif de la marchandise à l'acheteur par le vendeur.

Avec l'apparition de marchés organisés pour ces ventes à terme, favorisant la rencontre $d^{\prime}$ acheteurs et de vendeurs potentiels et garantissant les contrats contre la mauvaise foi de l'une des parties, des marchés « secondaires » se mirent en place où ces contrats purent être librement rachetés et revendus par des tiers entre le moment de leur création et celui prévu pour la livraison.

En fait, la création de marchés secondaires pour les opérations à terme ouvrit une boîte de Pandore : elle autorisa désormais la présence sur ces marchés d'intervenants n'ayant aucune intention ni de livrer ni de prendre livraison de la marchandise et dont le seul but est de parier sur la variation du prix entre le moment où ils interviennent sur le marché et celui prévu pour la livraison. Ces passagers clandestins, qui s'esquivent lorsque le moment de la livraison approche, ce sont bien sûr les "spéculateurs ». Les traders débutants, mandatés par un établissement financier pour spéculer sur ces marchés, commettent souvent l'erreur classique consistant à se dégager trop tardivement et se voient alors obligés de trouver en catastrophe des tonnes de carcasses de porc ou de graines de soja, ou, pire encore, de devoir en prendre livraison. La raison pour laquelle les spéculateurs sont tolérés sur les marchés à terme n'est pas celle que l'on entend toujours invoquer : leur capacité à offrir de la « liquidité ». Elle est en fait beaucoup plus simple : ces marchés sont dominés et tenus en main par les établissements financiers qui spéculent en leur sein au nom de leurs clients ou pour leur propre compte. Interpellés par ceux qui remettent en question la légitimité de leur présence, leur réponse est très simple : ce furent les intervenants légitimes - ceux qui sont présents sur ces marchés parce qu'ils entendent véritablement y acheter ou y vendre à terme une marchandise - qui les y ont invités parce qu'ils assurent une fonction essentielle à une mécanique bien huilée des marchés à terme : ils y assurent de la « liquidité ».

L'histoire est rapportée ainsi : pris d'une lubie soudaine, et agissant contre leur intérêt bien compris, les intervenants légitimes voulurent un jour interdire l'accès d'un marché à terme aux spéculateurs (le marché à terme de l'oignon dans les années 1950 , répète-t-on à voix basse) et mal leur en prit : le ciel leur tomba aussitôt sur la tête. Conscients de leur tragique erreur, et bourrelés de remords, ils invitèrent humblement les spéculateurs à les rejoindre. Ceux-ci dispensent désormais avec largesse - et sans manifester la moindre rancune - la manne de la liquidité dont ils sont les porteurs.

John Kenneth Galbraith - qui avait très mauvais esprit - disait à propos de la liquidité procurée par les spéculateurs qu'elle: «... favorise ce degré supplémentaire d'activité qui transforme un marché maigre et anémique en un marché gras et en bonne santé. II s'agit là dans le meilleur des cas d'un sous-produit qui ne fait pas grande différence et de toute manière d'un intérêt douteux » [2].

De manière plus générale, la seule liquidité qui compte pour un marché à terme de matières premières est celle de la marchandise ellemême : la capacité de la livrer ou d'en prendre livraison, la faculté d'en constituer des simulacres temporaires est indifférente.

Pour celui qui dispose réellement d'une marchandise ou qui la convoite, le marché à terme fonctionne comme une assurance. Pour celui qui n'en dispose pas, le marché à terme propose un casino. Contenant leur gratitude envers les spéculateurs et la manne de liquidité que ceux-ci procurent dans leur munificence et conscients du fait que leur présence massive signifie la mort certaine d'un marché - les organisateurs de marchés à terme ont généralement cherché à restreindre leur influence en limitant le nombre de contrats qu'ils sont autorisés à détenir.

Les spéculateurs considérèrent ces quotas comme une exigence excessive et ils mirent leur génie au service de la découverte de moyens de contourner cette barrière. C'est ce génie qui, en 2008, permit au prix des céréales et du pétrole d'atteindre dans le monde des sommets vertigineux : pareils au loup se parant de la peau d'une brebis, les spéculateurs, représentés par les banques d'investissement de Wall Street, envahirent les marchés à terme en se déguisant en "commercials », en intervenants légitimes, cherchant véritablement à vendre ou à acheter une marchandise. La CFTC (Commodity Futures Trading Commission), le régulateur américain des marchés à terme, en I'occurrence plus benête que malveillante, $n^{\prime} y$ vit que du feu, ouvrant la voie à une chaîne d'événements dont l'ultime aboutissement furent les émeutes de la faim du printemps 2008, provoquées par un renchérissement dramatique du prix des céréales sur les marchés mondiaux.

\section{L'entourloupe}

L'entourloupe qui a permis à la spéculation de jouer un rôle renouvelé sur les marchés à terme des matières premières fut révélée lors d'une réunion du Agricultural Advisory Committee qui eut lieu à Washington le 6 décembre 2007. Le mérite d'avoir extrait de la gangue des 276 pages de la transcription des auditions qui eurent alors lieu, leur substantifique moelle, revient à Michael Masters, qui en fit la synthèse le 20 mai 2008 devant le comité on Homeland Security and Governmental Affairs du Sénat américain [3].
Voici, expliqué brièvement, le mécanisme de l'entourloupe, destinée, je le rappelle, à neutraliser les quotas appliqués au nombre de spéculateurs sur les marchés à terme de matières premières. Faisant preuve - comme toujours $d^{\prime}$ une remarquable inventivité en matière d'ingénierie financière, les banques d'investissement de Wall Street contractèrent avec ceux que l'on appelle les « investisseurs institutionnels ", les fonds de pension, fondations universitaires américains, fonds souverains, etc., des swaps où ils promettent de verser à ceux-ci des sommes correspondant aux gains observés sur les marchés à terme des matières premières.

Si ces investisseurs institutionnels avaient opéré sur les marchés à terme sous leur propre identité, leurs positions auraient été limitées en taille. En se plaçant de manière indirecte, par le truchement d'un swap contracté avec une banque d'investissement, ils contournaient cette limitation.

Voici comment fonctionne ce swap : l'investisseur institutionnel s'engage à verser à échéance fixe, à la banque d'investissement, une somme correspondant au cours, à la date du contrat, d'un indice représentant la valeur d'un panier de contrats à terme de matières premières. La banque verse elle, à la même échéance, la valeur actuelle de cet indice. Les versements s'annulent bien entendu partiellement et à chacune des échéances, l'opération équivaut à ce que la banque verse à l'investisseur le gain de l'indice si celui-ci a crû entretemps, alors que l'investisseur verse le différentiel à la banque, si l'indice $a$, au contraire, décru au cours de la période.

Après avoir contracté de tels swaps avec une multitude d'investisseurs institutionnels, les banques d'investissement se tournèrent vers la CFTC en lui disant: "Las! Je suis exposé à un abominable risque sur le marché des matières premières: si le prix monte, je suis mort! Autorisez-moi - je vous en prie - à me couvrir contre ce risque abominable!». La CFTC, au bord des larmes, répondit en substance (selon le témoignage de Mr. Heitman, le 6 décembre 2007) : " Pas de problème puisqu'il s'agit de la couverture d'un risque! Allez-y, et pour faire bonne mesure, on vous attribuera le statut de "commercial" (= non-spéculateur)».

$C^{\prime}$ est de cette manière que les marchés à terme se firent rouler : les investisseurs institutionnels ne connurent désormais plus aucune limite à leurs positions spéculatives - puisqu'elles se contentaient de contracter des swaps avec les banques d'investissement de Wall Street, tandis que celles-ci avaient obtenu carte-blanche sur les marchés à terme où - selon la version officielle - elles n'ont jamais cherché qu'à se couvrir contre un abominable risque. 


\section{Spéculation et liquidité}

Revenons sur cette notion que la contribution positive qu'apporteraient aux marchés, les spéculateurs, ce serait la liquidité. En l'absence de spéculateurs, un marché risque, comme le dit Galbraith, d'être « maigre et anémique. » Leur présence assure, de l'activité, du volume. Grâce à leur présence, vendeurs comme acheteurs trouveront aisément, sur l'autre versant du marché, une contrepartie. Mieux, cette liquidité garantit un faible slippage, un faible « glissement » par rapport au prix recherché. En effet, en l'absence de liquidité, un vendeur devra fractionner le lot de titres ou de contrats qu'il cherche à vendre et finira par le vendre à différents niveaux de prix, et de même bien entendu pour l'acheteur qui se trouve en face et qui voudrait lui aussi acheter à un certain cours. Le slippage peut réduire considérablement le bénéfice d'une opération qui apparaissait prometteuse «sur le papier ». La liquidité qu'apportent les spéculateurs constitue donc un élément très positif pour les marchés.

... du moins en théorie ! Ce que je viens de dire semble à première vue hautement vraisemblable mais ne résiste pas à l'observation banale que les spéculateurs - et eux bien davantage encore que tout autre type $d^{\prime}$ acteurs présents sur les marchés - s'y trouvent dans le but unique de gagner de l'argent. Or, on gagne de l'argent en vendant plus cher que I'on n'a acheté, ce qui signifie que I'on achète quand le prix monte et que l'on vend quand le prix baisse. On suit donc la tendance générale. On peut bien entendu également faire I'inverse : vendre quand le prix monte et acheter quand il baisse, c'est ce qu'on appelle une stratégie " contrariante » et cela se rencontre beaucoup plus rarement parce que cela exige une connaissance exceptionnelle des marchés, permettant de déceler le moment exact du retournement : celui où le prix a tant grimpé qu'il ne peut plus que chuter et celui où il a tant baissé qu'il ne peut plus que remonter. À cela s'ajoute le fait que les marchés sont sélectifs : ils se débarrassent rapidement de ceux qui n'y comprennent rien. Bien sûr, le hasard joue aussi un rôle, il $n^{\prime}$ en reste pas moins vrai que celui qui est totalement privé de talent en ces matières doit compenser son handicap par une veine inouïe.

Les spéculateurs qui ne sont pas rapidement éliminés ont donc tendance à se retrouver groupés sur un seul versant du marché : celuiacheteur ou vendeur - en train de gagner, ce qui veut dire que la liquidité qu'ils sont censés procurer n'est pas nécessairement présente à I'appel, surtout si l'on se trouve - comme eux sur le versant gagnant. De même, la protection contre le slippage peut se révéler elle aussi illusoire, absente en tout cas lorsque son besoin se fait le plus ressentir: lorsque le marché s'emballe à la hausse ou à la baisse. Dans ces moments-là en effet les spéculateurs (à l'excep- tion des très rares « contrariants »), semblables aux passagers inexpérimentés d'un bateau dans la tourmente, se ruent tous du même côté du navire, précipitant son chavirement, c'est le krach, ou la bulle qui sera suivie d'un krach.

Compensant quelque peu ce que je viens dire, le fait que les spéculateurs possèdent des horizons de vente et $d^{\prime}$ achat différents et que le profil d'une heure ou d'une journée de trading peut aller à l'encontre de la tendance générale d'une semaine ou d'un mois. Les traits " suivistes» caractérisent cependant la nouvelle génération de spéculateurs dont Mike Masters a débusqué la présence : les investisseurs institutionnels présents sur le marché à terme des matières premières. Ceux-ci ne se trouvent en effet en permanence que sur un seul versant du marché : son versant « acheteur » où ils se placent dans la durée en «roulant» leurs positions d'une échéance sur la suivante. Ceci leur permet de se protéger contre l'inflation du prix des matières premières en achetant - par le truchement d'un indice et, comme je l'ai expliqué, grâce à l'astuce d'un swap - un panier de celles-ci.

Comme le fait finement observer Masters, ces «spéculateurs d'indice " n'ont pas même l'excuse de procurer au marché de la liquidité : présents sur le seul versant " acheteur », ils se contentent d'en consommer. Ils se positionnent sur un marché pour une somme particulière, disons 2 milliards de dollars, sans se préoccuper de savoir quelles sont les 25 matières premières qu'ils achètent de cette manière c'est l'indice qui s'est occupé de l'allocation - ni de quel est leur prix actuel - puisqu'ils le jouent à la hausse, les yeux fermés. Ils sont à ce titre parfaitement imperméables aux fluctuations de l'offre et de la demande. Masters observe :

"Si l'OPEP extrait davantage de pétrole cela aura très peu d'effet sur la demande des spéculateurs d'indice. Si les Américains réduisent leur consommation par un usage coopératif des véhicules ou en recourant davantage aux transports en commun, cela aura également très peu d'impact sur leur demande. Les stratégies des investisseurs institutionnels sur les marchés à terme des matières premières s'assimilent à du stockage virtuel... "

Les investisseurs institutionnels contribuent ainsi unilatéralement à l'augmentation des prix : plus le prix des matières premières monte plus leur stratégie visant à se protéger contre I'inflation résultant de la hausse du prix de ces produits se trouve justifiée et plus ils augmentent leur mise. Le fait que ce soit leur comportement moutonnier qui est à la source de cette inflation ne constitue pas à leurs yeux une objection recevable : on les imagine aisément rétorquer : "Quoi, je serais le dernier à me protéger! » On trouve ici une confirmation de plus qu'aucune "main invisible » ne contribue à faire émerger le bien général de la poursuite insatiable des intérêts particuliers.

\section{Spéculation et prix}

Les acheteurs et les vendeurs légitimes sur les marchés à terme des matières premières, ceux qui livrent une marchandise ou en prennent livraison, influent de la manière classique sur les prix : en fonction d'un rapport de forces qui est à certains moments favorable aux vendeurs (et le prix alors monte) et à d'autres, favorable aux acheteurs (et le prix alors baisse); c'est ce qu'on appelle traditionnellement «le mécanisme de l'offre et de la demande » bien que ce soit beaucoup plus compliqué que cela - beaucoup plus centré sur les acteurs eux-mêmes qu'une simple question d'offre et de demande. Les spéculateurs "classiques", suivent la tendance : ils achètent quand le prix monte et ils vendent quand il baisse. Ils sont donc neutres du point de vue de la formation du prix. Les spéculateurs d'indice, les investisseurs institutionnels américains sur lesquels Mike Masters a attiré l'attention et dont le volume sur les marchés à terme est passé de 13 milliards de dollars en 2003 à 260 milliards en mars 2008 - achètent uniquement et poussent donc uniquement à la hausse du prix $-+183 \%$ sur la même période.

Pour avoir une idée de la force respective des trois camps en présence, j'ai emprunté ses chiffres à Mike Masters ${ }^{3}$, et voici le graphique qui les représente. Les opérateurs légitimes sont en bleu, les spéculateurs classiques en bordeaux et les spéculateurs d'indice en jaune ; on observe que ces derniers représentent aujourd'hui à peu près le même poids que les opérateurs légitimes. On observe par exemple que, comme on l'imagine, les spéculateurs « classiques » sont très présents sur le marché à terme de l'or (figure 1).

Imaginons que l'offre augmente : 1 ) le facteur " offre et demande » pousse à la baisse du prix ; 2) le facteur «spéculateur d'indice " pousse à la hausse, comme il le fait en permanence. Tout dépendra si l'offre augmente suffisamment pour compenser la tendance à la hausse due aux spéculateurs d'indice. Les spéculateurs « classiques» viendront au renfort de la tendance qui émergera - quelle qu'elle soit. Conclusion : le prix ne baisse que si l'offre augmente de manière "significative», à savoir suffisamment pour compenser l'inflation constante due aux spéculateurs d'indice.

Si au contraire l'offre baisse: 1) le facteur « offre et demande » pousse à la hausse du prix ; 2) le facteur "spéculateur d'indice» pousse lui aussi à la hausse, comme à son habitude. Les spéculateurs « classiques » courent au secours de la victoire, et apportent le support de leurs troupes à la tendance pour le

\footnotetext{
$\overline{3}$ http://www.dpc.senate.gov/dpcnew.cfm?doc_name=lb-110-2-117
} 


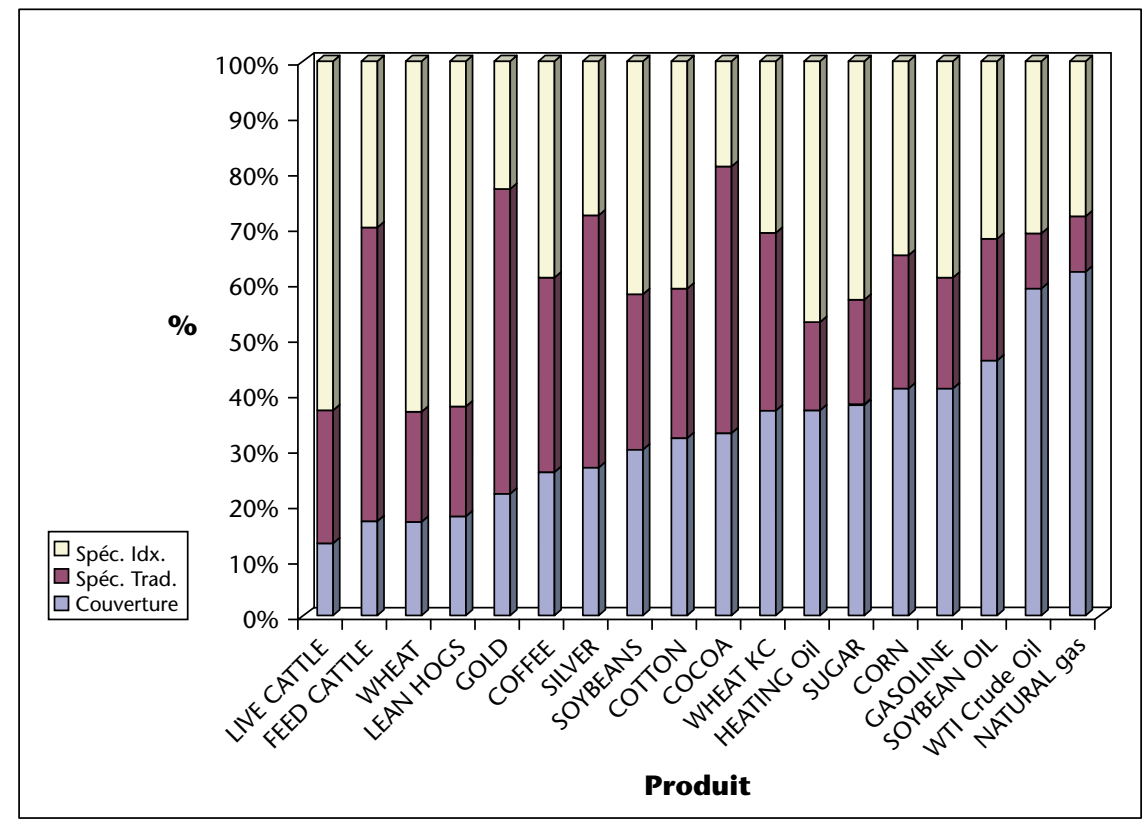

Figure 1. Acteurs présents sur le marché à terme de matières premières.

prix à s'élever. On observe les courbes grimpant « exponentiellement » que l'on a connues durant le premier semestre 2008.

Le pétrole joue ici un rôle particulier. Tout d'abord, l'augmentation de son prix se répercute indirectement sur l'ensemble de celui des produits agricoles - végétaux et animaux - du fait que des produits dérivés du pétrole sont impliqués dans leur production: gasoil, engrais, propane, pesticides, etc. Ensuite, l'augmentation de son prix a un impact direct sur celui du maïs utilisé désormais comme substitut du pétrole dans la production de bio- carburants : plus ce prix est élevé, plus l'éthanol lui devient un substitut compétitif. De plus, dans le contexte qui nous est familier, où toute augmentation de la productivité agricole est nécessairement lente et où les diverses céréales peuvent partiellement se substituer les unes aux autres, une augmentation du prix du pétrole n'a pas seulement une influence sur celui du maïs mais aussi sur celui de l'ensemble des céréales. Ce que je viens de dire ici est - on l'aura noté - entièrement indépendant de la question du «pic pétrolier » : ne joue que le fait que le prix du pétrole influence de manière directe (éthanol) ou indirecte (céréales, viande) le prix d'autres matières premières.

Deuxième graphique, celui-ci emprunté à l'exposé de Masters lors de sa déposition devant le Sénat américain : I'augmentation des volumes et des prix sur les marchés à terme des matières premières. Les prix ont manifestement crû et cette croissance à correspondu à l'irruption en masse des investisseurs d'indice, dont le volume est représenté par la surface du disque rouge (figure 2).

La figure 3, empruntée à un nouveau rapport de Mike Masters, diffusé celui-ci le 10 septem-

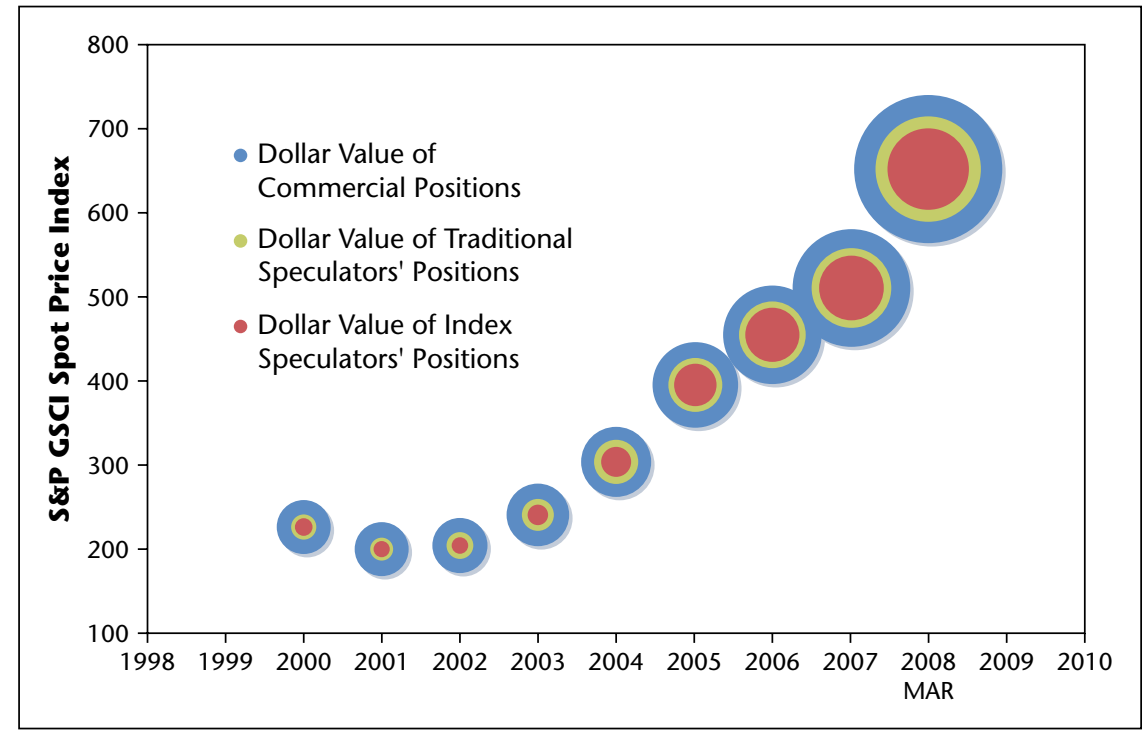

Figure 2. Augmentation des volumes et des prix sur les marchés à terme des matières premières. Source : Bloomberg, CFTC Commitments of traders CIT supplement, calculations. 


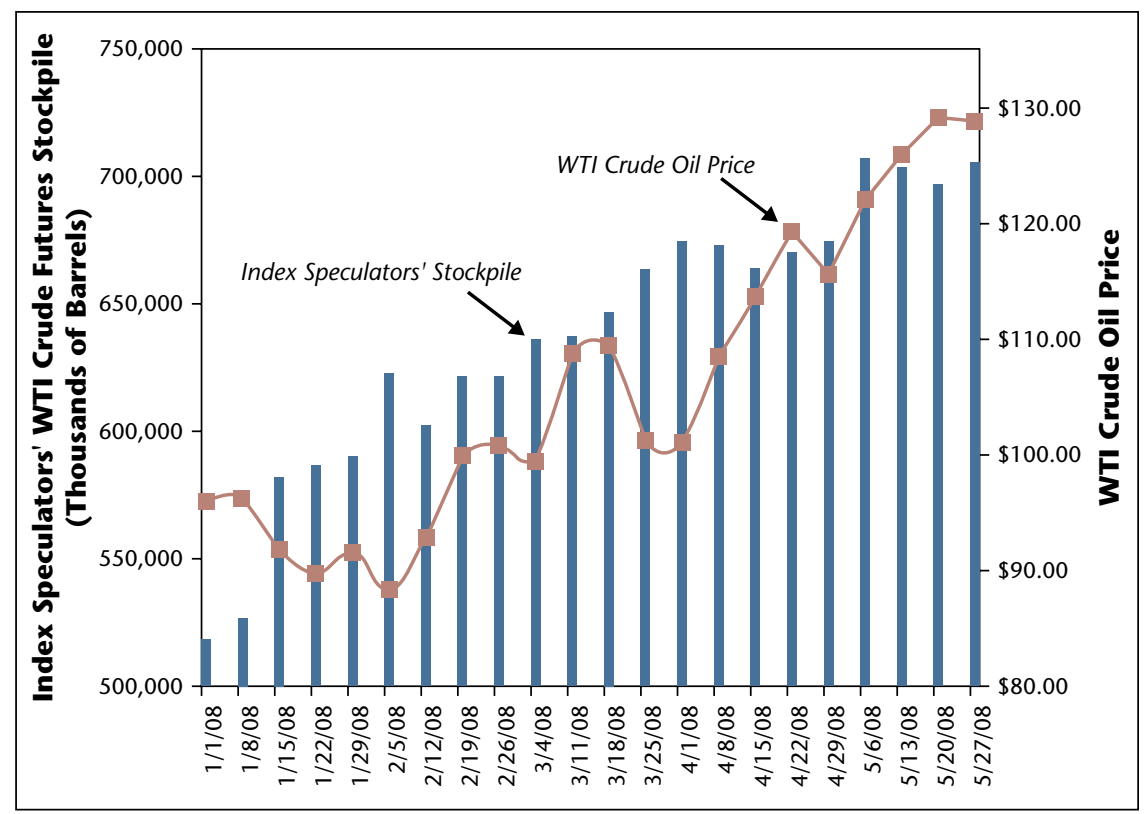

Figure 3. Prix du brut sur le marché à terme et volume des positions de swap sur l'indice à terme des matières premières.

Source: Bloomberg, Standard and Poors, Dow Jones, calculations based upon the Commodities futures trading commission's commodity index trader report.

bre [4], confirmait ceci en mettant en parallèle de prix du brut sur le marché à terme et le volume des positions de swap sur l'indice à terme des matières premières

Aucun calcul de « covariation » entre les deux courbes n'est nécessaire : la coïncidence des tendances est clairement visible.

Ayant dit cela, il n'était bien sûr pas certain que les spéculateurs d'indice persisteraient à parier contre vents et marées sur la hausse des prix.
Pourquoi l'ont-ils fait de 2003 à juillet 2008 ? Pour se protéger contre l'inflation. Ils se protégèrent contre la déperdition de valeur des matières premières exprimées en dollars en plaçant leurs dollars dans des contrats portant sur ces marchandises qui s'apprécient si leur prix monte. C'est une stratégie classique de " couverture " : à mesure que le dollar perd de sa valeur par rapport aux autres devises, le prix de ces matières premières augmente, et cette augmentation compense la baisse de la devise, si bien que l'on s'y retrouve au bout du compte. Bien sûr, c'est une prophétie autoréalisante : cette inflation que les spéculateurs $d^{\prime}$ 'indice redoutent, ils contribuent à la créer, mais ça, ce n'est pas leur affaire! Que fallait-il pour que ces spéculateurs d'indice soit se retirent des marchés à terme des matières premières, soit parient cette fois sur leur baisse ? II fallait bien sûr que la tendance se renverse. Une

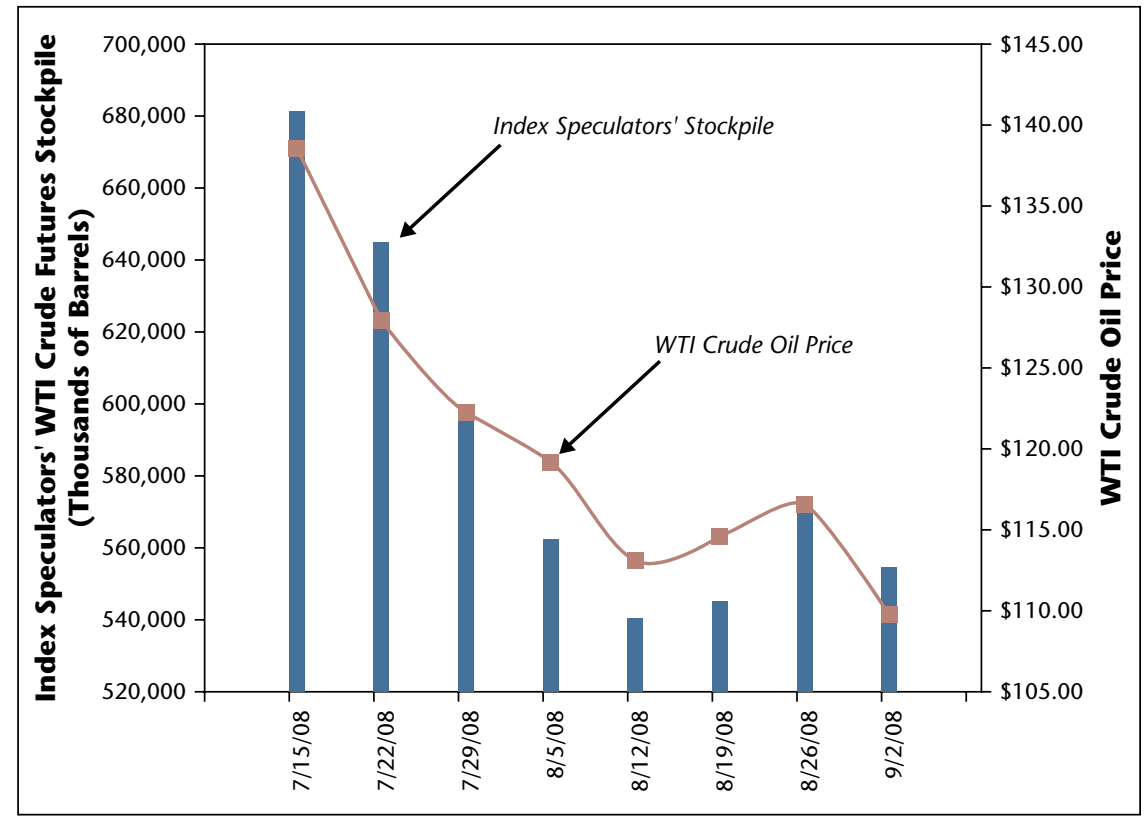

Figure 4. Montant des investissements dans les indices à terme de matières premières superposé au prix à terme du pétrole. Source : Bloomberg, Standard and Poors, Dow Jones, calculations based upon the Commodities futures trading commission's commodity index trader report. 
possibilité, c'était que le dollar s'apprécie par rapport aux autres devises : le prix des matières premières exprimé en dollars deviendrait alors relativement bon marché et il faudrait alors parier à la baisse. Une autre possibilité - due à la position particulière du pétrole dans l'équation - était que l'offre du brut augmente suffisamment pour contrer la pression inflationniste des spéculateurs d'indice orientés aujourd'hui à la hausse, augmentée de celle des spéculateurs classiques qui se contentent, eux, d'aller là où le vent les pousse.

Or ce qui se passa fut la conséquence d'un mécanisme encore plus simple: la peur du gendarme! Mike Masters fit sa déposition devant un comité du Sénat américain, le 20 mai 2008. Elle eut un énorme retentissement : il convainquit un nombre considérable $d^{\prime}$ 'hommes politiques et ce fut un branle-bas de combat immédiat : les projets de loi visant à brider la spéculation sur les matières premières fleurirent comme muguet en mai, dont le projet de loi Stop Excessive Energy Speculation Act of 2008 que j'évoquais plus haut.
Second graphique (figure 4), emprunté lui aussi au second rapport Masters, couvrant celui-ci la période du 15 juillet au 2 septembre [4]. Les spéculateurs se précipitèrent vers les issues de secours. Même convention dans la représentation : le montant des investissements dans les indices à terme de matières premières a été superposé au prix à terme du pétrole.

Les spéculateurs - de l'ancienne ou de la nouvelle engeance - contribuent donc à amplifier le mouvement des prix dans un sens ou dans l'autre: pour créer la bulle quand ceux-ci montent et pour précipiter le krach quand ils baissent.

La seule justification de leur présence sur les marchés était qu'ils y apportaient de la liquidité. On a vu ce qu'il fallait en penser.

Dans Une constitution pour l'économie: un exemple $^{4}$, je proposais un article rédigé ainsi :

${ }^{4}$ elsevier.com/xml/linking-roles/text/html" xlink:href="http://www.pauljorion.com/blog/ $? p=407$ "http://www.pauljorion.com/blog/?p=407
«Les ventes à terme ainsi que les options $\mathrm{d}^{\prime}$ achat sont autorisées. Elles lient leurs contreparties jusqu'à livraison. En conséquence, il n'existe pas pour elles de marché secondaire. Les paris relatifs à l'évolution d'un prix sont eux interdits».

Mike Masters m'a donc offert tous les arguments - et tous les chiffres - dont j'avais besoin pour soutenir ma proposition.

\section{RÉFÉRENCES}

1. SCHWAGER JD. The New Market Wizzards. Conversations with America's top traders. New York : HarperBusiness, 1992.

2. GALBRAITH JK. The Great Crash - 1929. Boston : Houghton Mifflin, 1954.

3. MASTERS M, WHITE AK. The Accidental Hunt Brothers. How Institutional Investors Are Driving Up Food and Energy Prices. Special Report July 312008.

4. MASTERS M, WHITE AK. The Accidental Hunt Brothers - Act 2. Index Speculators Have Been a Major Cause of the Recent Drop in Oil Prices. Special Update September 102008. 